AperTO - Archivio Istituzionale Open Access dell'Università di Torino

MET/HGF pathway in multiple myeloma: From diagnosis to targeted therapy?

This is a pre print version of the following article:

Original Citation:

Availability:

This version is available http://hdl.handle.net/2318/1527050

since 2015-10-29T08:31:34Z

Published version:

DOI:10.1586/14737159.2015.1046436

Terms of use:

Open Access

Anyone can freely access the full text of works made available as "Open Access". Works made available under a Creative Commons license can be used according to the terms and conditions of said license. Use of all other works requires consent of the right holder (author or publisher) if not exempted from copyright protection by the applicable law. 


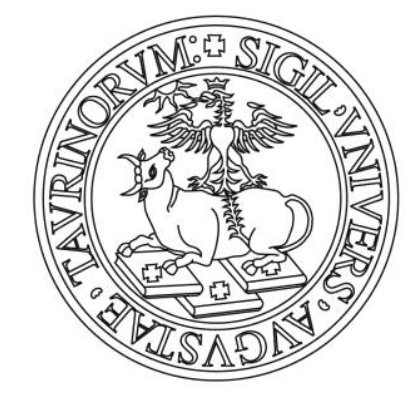

\section{UNIVERSITÀ DEGLI STUDI DI TORINO}

This is an author version of the contribution published on:

Questa è la versione dell'autore dell'opera:

Expert Rev Mol Diagn. 2015;15(7):881-93. doi: 10.1586/14737159.2015.1046436

The definitive version is available at:

La versione definitiva è disponibile alla URL:

http://www.tandfonline.com.offcampus.dam.unito.it/doi/full/10.1586/14737159.20

15.1046436 


\title{
MET/HGF pathway in multiple myeloma: from diagnosis to target therapy?
}

\author{
Manuela Gambella ${ }^{1}$, Antonio Palumbo ${ }^{1}$, Alberto Rocci ${ }^{2}$ \\ ${ }^{1}$ Myeloma Unit, Division of Hematology, University of Torino, Azienda Ospedaliero-Universitaria Città della \\ Salute e della Scienza di Torino, Italy \\ ${ }^{2}$ Department of Haematology, Manchester Royal Infirmary hospital, Oxford Road, Manchester, UK
}

\begin{abstract}
Interaction between neoplastic cells and the microenvironment is critical in several cancers and plays a central role in multiple myeloma. Microenvironmental stimuli support plasma cells proliferation, survival, motility and can determine drug resistance. The network between plasma cells and surrounding cells is also responsible for increasing angiogenesis, unbalancing bone formation and bony lesions. The MET/HGF pathway is a key player of this interaction and has been found to be abnormally active in both malignant plasma cells and surrounding cells. Patients with abnormal MET and/or HGF levels usually experience a poor outcome even when treated with novel drugs. This review addresses the role of MET/HGF in the pathogenesis of myeloma and describes the role of MET/HGF signaling as a prognostic factor. The different techniques to detect MET/HGF abnormalities will also be examined. A final discussion on compounds targeting MET/HGF will summarize the current opportunities to introduce targeted therapy in myeloma patients.
\end{abstract}

Key Word: myeloma, MET, HGF, target therapy, prognosis

Conflict of Interests: Gambella: no COI. Palumbo Consultancy fees: Amgen, Bristol-Myers Squibb, Genmab A/S, Celgene, Janssen-Cilag, Millennium Pharmaceuticals Inc., Onyx Pharmaceuticals. Honoraria: Amgen, Novartis, Bristol-Myers Squibb, Genmab A/S, Celgene, Janssen-Cilag, Millennium Pharmaceuticals Inc, Onyx Pharmaceuticals, Sanofi Aventis. Rocci: no COI.

Acknowledgements: The authors would like to thank Dr. Livio Trusolino for the critical review of the draft. 


\section{The MET/HGF pathway}

The interaction of malignant cell with the microenvironment is a key feature of many cancers, determining growth, drug resistance and invasion. The network between different cells is based on both cell-to-cell interactions and soluble factors. The latters are produced in one cell and act paracrinally on neighboring ones, permitting change of information also in cells without a direct contact.[1] The role of the microenvironment is pivotal in multiple myeloma $(\mathrm{MM})$, a blood cancer generating from bone marrow (BM) plasma cells (PCs) characterized by abnormal relationships with stromal cells, osteoclasts, osteoblasts and endothelial cells.[2] The Hepatocyte Growth Factor (HGF) is a key component of the network between different BM cells.

HGF is a $90 \mathrm{~K}$ multidomain glycoprotein physiologically produced and secreted by mesenchymal cells as a single-chain precursor (pro-HGF), which is then proteolytically cleaved to biologically active heterodimeric molecules by enzymes such as the serine protease HGF activator (HGFA).[3] The mature form consists of an $\alpha$-chain (with a $\mathrm{N}$-terminal domain and 4 kringle domains) and a $\beta$-chain (Serine proteases homology domain) linked by a disulfide bond. HGF is a potent angiogenic cytokine with ability to induce proliferation and migration of endothelial cells (EC).[4] In PCs HGF is strictly combined with surface protein CD138, the hallmark of myeloma cells. CD138 binds HGF and presents higher concentration of this factor to its receptor MET.[5] Physiologically HGF is able to modulate bone production/absorption and to regulate angiogenesis both directly and through modulation of other cytokines.

The receptor of HGF is the tyrosine kinase (TK) MET, a surface protein encoded by a gene located on chromosome 7q21-31.(Fig.1) It has an extracellular $\alpha$-chain linked to a transmembrane $\beta$-chain by a disulfide bridge. The extracellular portion is composed of three domain types: the Sema domain, a key structure for receptor binding; the plexin-semaphorinintegrin (PSI) domain; and 4 immunoglobulin-plexin-transcription (IPT) domains. The extracellular part is linked to the intracellular portion that includes: a juxtamembrane region containing serine residues (S975, responsible for inhibition of MET activity) and tyrosine residues (Y1003, responsible of MET degradation); a catalytic region containing two catalytic tyrosines (Y1234 and Y1235, modulating MET enzymatic activity); a C-terminal region with two "docking" tyrosine residues (Y1349 and Y1356, able to bind SH2-containing intracellular effectors).[6]

Following the binding of HGF with MET, a dimerization of the receptor leads to the transphosphorylation of $\mathrm{Y} 1234$ and $\mathrm{Y} 1235$ (which increases the intrinsic catalytic activity of the kinase) followed by auto-phosphorylation of Y1349 and Y1356 (which recruits signal transducers to the receptor tail, thus activating different downstream pathways. The outcome of Met signaling includes several biological responses such as growth stimuli, reduction of apoptosis, increase in angiogenesis, ability to migrate and to infiltrate surrounding tissues. [7] 
Several adaptor proteins and kinase substrates are involved in transducing MET signal. The major substrate is considered to be Gab1, a multi-adaptor protein that can interact with MET both directly or via Grb2. This leads to the activation of several signaling cascade such as PI3K and AKT (promoting cell survival and migration) or Shp2 via Ras/ERK/MAPK (regulating proliferation and branching morphogenesis).[8-9] Similarly, the activation of Crk with Rap1 and Rac can mediate cell motility and brancingh morphogenesis.[10] MET can also induce VEGF expression via recruitment of Shc.[11]

Physiologically, the MET/HGF pathway has a crucial role in morphogenesis, while in the adult is involved in the development and regeneration of epithelial organs.[6] The MET/HGF pathway has also been implicated in development of hematopoietic cells; in fact, MET is expressed by a subset of hematopoietic precursor cells, whereas HGF is expressed by the bone marrow stromal cells (BMSCs).[12-13] Under normal conditions, MET/HGF activation is tightly regulated through paracrine ligand delivery, ligand activation at the target cell surface, and ligand-activated receptor internalization and degradation.[14] However, a deregulation of MET/HGF signalling is implicated in oncogenesis and progression of different solid malignancies, including colon, gastric, breast, ovarian, pancreatic, and lung tumors.[15-17] Aberrant MET/HGF pathway has also been implicated in the development and progression of $B$ cell malignancies, including MM.

Aberrant MET signaling can occur through different mechanisms, such as gene amplification, mutations, overexpression, excessive autocrine or paracrine ligand-mediated stimulation and interaction with other active cell-surface receptors.[18-19] In details:

MET gene amplification: it causes overexpression of MET and has been reported in a number of human primary tumors, including gastric and esophageal carcinomas, medulloblastomas and liver metastases from colon carcinoma. In many of these cancers, patients carrying MET amplification showed a poor response to treatment and a dismal prognosis. In Non-Small Cell Lung Cancer (NSCLC) the presence of MET amplification causes resistance to EGFR inhibitors and characterizes patients with worst outcome.[20]

MET mutations: mutations have been detected across the different MET domains, in particular its TK domains, deregulating either the activation or the degradation of the receptor. Several activating missense mutations of the MET gene have been identified as determining an aberrant signaling cascade in sporadic and hereditary papillary renal cancer, hepatocellular carcinoma, and gastric cancer.[21]

MET overexpression: can be a result of transcriptional up-regulation due to upstream gene mutations or can be caused by hypoxia, inactivation of tumor suppression genes or loss of microRNAs.[3-22-23] MET overexpression can also be due to MET gene amplification. High levels of MET on the cell surface can lead to ligand-independent activation through spontaneous dimerization and phosphorylation.

Abnormal HGF production: can be due to cancer cells or surrounding cells, sustaining cell growth in an autocrine or paracrine manner respectively. These mechanisms are present in 
MM as well as in many other cancers. HGF production can be further stimulated by hypoxic conditions via HIF-1a.[24]

\section{MET/HGF in the pathogenesis of multiple myeloma}

$\mathrm{MM}$ is the second most frequent blood cancer and derives from PCs, the late differentiation stage of B lymphocytes. Clonal PCs accumulate in the BM, produce intact (paraprotein) or partial (free light chain) immunoglobulines and are responsible for organ damage such as hypercalcaemia, kidney failure, anaemia and bony lesions. Many molecular alterations have been described in PCs, but very few of them have been considered as pathognomonic of MM. Chromosomal alterations (translocations involving the regulator of $\mathrm{lgH}$ locus and deletion of Ch13 and 17), mutations of oncogenes, abnormal expression of genes involved in lymphocyte maturation and miRNAs deregulation have been all involved in the pathogenesis and progression of MM.[25-26-27] Beside these, the signals produced by the microenvironment have always been considered as a key part of the disease, in particular of the progression from a premalignant phase to a symptomatic phase. Among all the cytokines stimulating MM growth, HGF is considered to be of pivotal importance because it is secreted both by PCs and BMSC.(Fig.2)

The first observation of a possible role of MET/HGF in MM was done by Börset et al. in 1996 investigating mRNA expression of HGF and MET in PCs and HGF protein content in the serum of MM patients. They observed that serum HGF levels were higher in MM patients than in healthy subjects (about 4 fold) and that MM PCs had concomitant expression of MET and HGF mRNA. This observation was peculiar of malignant PCs and was not found in PCs of MGUS patients, suggesting for the first time a possible autocrine loop sustaining MM cell growth.[28] Since then, several biological and clinical papers confirmed that aberrant expression of this pathway is common in MM. It was also shown that high serum levels of HGF activator (HGFA) are a distinguishing feature of BM of MM patients.[29-30]

Beside the concomitant expression on MET and HGF in MM PCs (autocrine loop), HGF is secreted by stromal cells as well, confirming the presence of a paracrine HGF/MET communication. Notably, myeloma cells and stromal cells produce both HGFA and HGF, so the latter is processed in its active form.[31] This is an important step in HGF/MET signaling, allowing the MM cells to regulate the availability of HGF. Wader and colleagues showed with immunohistochemical analysis that MET exists in its phosphorylated state in a relevant number of MM patients indicating that HGF/MET system is active in MM patients in vivo; furthermore, it is expressed only in malignant PCs and MET levels increase along with disease progression.[32] HGF interacts with other surface molecules such as the heparin sulfate proteoglycan syndecan 1 (CD138), a hallmark of MM PCs. The high expression of

Syndecan-1 on PCs increases HGF-mediated signaling, promoting cell survival and proliferation.[5] 
It has been clearly demonstrated that angiogenesis is increased in MM patients compared to patient with MGUS and it correlates with prognosis.[33] In agreement with the well known angiogenic properties, HGF signaling promotes angiogenesis and endothelial cell growth through direct interaction with MET (which is physiologically expressed in endothelial cells) or through an indirect cross-talk with the vascular endothelial growth factor (VEGF) and VEGF receptor (VEGFR) pathway. Indirectly, HGF can enhance the expression of VEGF and, similarly, can downregulate TSP-1 expression. The down-regulation of TSP-1 is mediated by different intracellular effectors such as MAPK, PI3-k or Stat3 according to the tissue analyzed. In addition to the MET/HGF loop found in the plasma cells, there is also a similar loop in the ECs of patients with MM and this can further modulate a network of cytokines resulting in enhancement of angiogenesis.[34]

The VEGFA/VEGFR2 autocrine loop has been found in endothelial cells (ECs) obtained from BM with active MM. Ferrucci et al. have shown that HGF/cMET pathway is only activated in multiple myeloma ECs of patients with active disease (diagnosis, refractory and relapse)[35]; moreover, the important increase of MET phosphorylation in ECs suggest that this pathway is constitutively activated in MM.[32] Therefore, the HGF/cMET autocrine loop may act as an additional angiogenesis amplifier for MM ECs. MET and HGF are expressed at a higher level in ECs of MM patient when compared with MGUS patients. This data has been confirmed at both mRNA and protein levels. As expected, MM ECs also secrete more HGF (2.5 fold on average) than MGUS EC as part of a self-sustaining loop.[35]

It was demonstrated that HGF promotes the adhesion and migration of myeloma cells in vitro, implying that HGF/MET signaling could also be involved in the extravasation and homing of MM cells in vivo.[36-37-38] Interestingly, it was shown that MET signaling enhances the actions of interleukin- 6 whose mechanism of action is the inhibition of MM cell apoptosis.[39] This suggests that MET signaling could also play a central role in MM biology by potentiating the effect of other myeloma growth factors.

The balance between bone formation and bone resorption is lost in many cases of MM, resulting in bone destruction and the development of osteolytic lesions. During the whole history of the disease, more than $80 \%$ of patients experience bony lesions determining severe impairment in quality of life. Several observations indicate that MET/HGF pathway has a role in bone metabolism and MM-associated bone lesions.[40]

MET is expressed on the osteoblast surface and HGF binding can inhibit osteoblastogenesis induced by bone morphogenetic protein (BMP). Standal et al. demonstrated that ALP (Alkaline Phosphatase, a specific marker of osteoblast activity) is reduced in vitro after HGF is added to the culture medium. HGF has been shown to also inhibit osteoblast-specific transcription factors Runx2 and Osterix and reduce the nuclear translocation of SMAD 1,5,8. These in vitro findings have been confirmed in biological samples of MM patients: a negative correlation has been found between HGF and ALP levels, supporting the role of HGF as an 
inhibitor of osteoblastogenesis.[41] mRNA HGF levels are also increased in MM PCs of patients with MM when compared with MGUS patients, and directly correlate with the presence of lytic bone lesions. Both MET and HGF mRNA were high in patients with advanced lytic bone lesions and a similar pattern was observed at the protein level, confirming in vivo the strong correlation between HGF and lytic lesions. [42] HGF has also been found to be produced by osteoclasts while MET receptor is located both at the surface of both osteoclasts and osteoblasts.[43] These observations suggest the possibility of an autocrine stimulation within osteoclasts and a concomitant paracrine stimulation in osteoblasts. Moreover, HGF stimulation induces IL-11 secretion from osteoblasts, which acts by stimulating osteoclast formation and suppressing bone formation.[44] Collectively, all these findings support the notion that the HGF/MET system contributes to bone erosion, while impairing bone formation.

\section{MET/HGF as prognostic factor in myeloma}

The observation that high HGF levels in serum of MM patients were able to predict poor response to treatment and short outcome was first reported more than 15 years ago.[45] Sera from 398 patients treated with MP were analyzed: 60\% of patient with low HGF levels achieved a plateau phase, compared with only $46 \%$ of patients with high HGF and $27 \%$ of patients with extremely elevated HGF levels. This trend was confirmed on OS, with median OS of 32, 21 and 11 months for patients with low, high and very high HGF levels respectively. Similarly, the overexpression of MET mRNA in PCs of MM patients was also associated with low response to therapy and poor clinical outcome.[46] We investigated MET mRNA expression in 105 newly diagnosed MM patients treated with bortezomib-based therapy. The crude analysis of clinical outcome in the group of patients with high vs low MET mRNA expression (median value was used as a cutoff) showed a better prognosis in patients with low MET expression. In particular, the 4-year PFS was 39\% in patients with low MET vs $20 \%$ in those with high MET and similarly the OS was $77 \%$ vs $51 \%$. Interestingly, MET expression was independent of any other parameters used as prognostic factors in MM and these results were confirmed when MET expression was combined with $\beta 2$-microglobulin: patients with both high MET mRNA and high $\beta 2$-microglobulin had worst PFS and OS ( $8 \%$ and $32 \%$ ) compared with the other patients ( $38 \%$ and $76 \%$ respectively). No differences were observed when patients were divided according to the mRNA expression of HGF. In 82 patients we were also able to quantify the MET copy numbers using FISH analysis. We have not found any monosomy of MET gene but in 30 patients (37\%) we identified 3 or more copies of MET gene. There was no difference in MET mRNA expression values according to gene copy numbers, but patients carrying 4 copies $(n=8)$ had a shorter survival compared to the other patients. The small numbers can only suggest copy number gains as a mechanism of growth advantage, thus underlying a more aggressive disease; however the independency of MET copy number and MET expression suggests that both these mechanisms can be implicated in determining partial response to therapy and poor outcome. 
MET expression was found to be higher in MM than in MGUS but also higher in relapsed MM than in newly diagnosed MM. This implicates MET expression as a characteristic of recurrence and possibly as a causative event in progression of MM.[35] To confirm the link between HGF levels and MM, it was observed that HGF bone marrow plasma levels decrease with successful treatment responses and the serum levels of soluble form of MET (a negative regulator of HGF/MET activity) negatively correlate with disease stage.[47-48]

\section{MET/HGF and drug resistance}

MM is an incurable disease in most of the patients and the aggressiveness of PCs increases at each relapse. In fact drug resistance is the ultimate cause of disease progression due to the refractoriness to available treatments. MET has been described to be implicated in determining resistance to chemotherapy in several cancers including MM.[49-50] Several reports are suggesting that MET can be involved in drug resistance to therapy specifically targeting EGFR or angiogenesis; for instance, in EGFR mutant non-small cell lung carcinoma (NSCLC) amplification of MET was observed in $22 \%$ of tumors resistant to EGFR inhibitors.[20]

The MET pathway is overexpressed in MM cell lines showing drug resistance as well as in primary samples from patients with relapse/refractory MM.[51] It has been reported that multidrug resistance MM cell line R5 is characterized by high levels of MET mRNA, MET protein and phospho-MET protein level. These findings are typical of the R5 cell line and are different from the chemo sensitive cell line RPMI-8226, which features normal MET levels. The authors also evaluated the role of SU11274, a highly specific MET inhibitor, in overcoming drug resistance in MM cells. SU11274 is able to specifically inhibit HGF-induced phospho-MET and its downstream signal.[52] Moschetta et al. tested the effects of SU11274 in both cell lines and the reduction in phospho-MET was maximal in R5 while it was weak in RPMI-8226. This finding was observed also in another multiresistant cell line, MM.1R, compared to the sensitive cell line MM.1S. SU11274 determined a potent apoptosis in R5 cell lines (7 fold higher apoptosis compared to DMSO) but not in RPMI-8226 (only 2 fold higher). SU11274 was also able to overcome drug resistance to bortezomib, doxorubicine and melphalan in R5 cells. A similar observation was reported earlier with the U266 cell line, in which the knockdown of MET using small-interfering RNA (siRNA) increased cell sensitivity to chemotherapy agents.[53-54] The efficacy of SU1274 was confirmed in vivo in xenograft mouse models: a higher efficacy of the compound was observed in mice injected with the resistant R5 cell linethan in mice bearing xenografts of sensitive RPMI-8226. Trying to translate these observations into the clinical arena, authors treated primary 138+ cells with SU11274. The results showed more substantial effects in cells from relapsed/refractory MM patients compared with newly diagnosed ones, in line with the observation that phospho-MET was higher in the first group than in the latter. 
The MET pathway has also recently been involved in carfilzomib resistance. Carfilzomib is a second-generation irreversible proteasome inhibitor recently introduced in the armamentarium of anti-MM compounds. Wang et al. generated several carfilzomib-resistant MM cell lines and investigated the differences in gene expression profiles between the wild type and the resistant cell lines. They found that suppression of MUC20 was consistently present in carfilzomib-resistant cell lines and was also a characteristic of bortezomib-resistant cell lines. The reduced expression of this gene (and protein) determines an increase in phospho-MET with activation of downstream signaling via STAT3 and ERK1-2. Thus the indirect activation of the MET pathway is one of the mechanisms likely responsible for resistance to either bortezomib or carfilzomib. When a MET inhibitor was used in combination with carfilzomib, it was able to restore carfilzomib sensitivity in previously resistant cell lines and in a murine model. A further validation to this is the observation that patients with high levels of MUC20 had a better outcome (PFS and OS) than patients with low MUC20 levels treated with bortezomib.[55]

These results have been confirmed in vivo by our study, showing that low MET mRNA expression in PCs identified patients with a better response to bortezomib-based treatment.[46] A consistent increase in MET expression has been observed in patients achieving a CR compared with those exhibiting stable disease. Median MET mRNA expression was 56.10 in patients achieving at least a VGPR but was 134.83 in those achieving only a PR or a SD. This finding suggests that different expression levels of MET can predict drug sensitivity and finally response to therapy.

\section{Techniques to evaluate MET/HGF in the diagnostic process}

Many studies have determined plasma and serum levels of HGF protein using an enzymelinked immunosorbent assay (ELISA) kit. Wader et al. have used this detection method also to determine the serum and plasma levels of a soluble form of MET in patients with MM (Wader et al. Eur J Haematol 2011). The serum and plasma samples are previously recovered and stored at $-80^{\circ} \mathrm{C}$ and thawed at the moment of the analysis. Many samples can be analyzed in a single batch on the same day and a standard curve is generated for each set of samples assayed.[56-57]

The distribution of HGF and MET protein in biopsies is usually investigated by immunohistochemistry (IHC). In IHC the starting materials are biopsies previously fixed in formalin, decalcified in ethylenediamine tetraacetic acid (EDTA) and embedded in paraffin wax. The immunohistochemical evaluation is performed using specific antibodies against MET, phospho-MET or HGF. The Nordic group has examined the expression of HGF and MET in 80 biopsies from patients with monoclonal plasma cell disease; only approximately $10 \%$ of biopsies were not evaluable because high background. The biopsies evaluable 
showed a cytoplasmic staining for HGF in PCs while a membranous and/or cytoplasmic staining for MET was detected. However, there may be hurdles in discriminating a weak cytoplasmic/nuclear staining due to high background: indeed, the interpretation of sections with limited intensity and extent of staining may prove difficult when scoring positive versus negative samples.[32-42] In IHC the expression of MET is a hallmark of MM PCs and it is absent in PC of healthy subjects or in MGUS patients. This specific MET expression can be useful in distinguishing malignant from non-malignant PCs.

MET, phosphor-MET and HGF can also be assessed using western blot: the cell lysates are probed with primary antibodies and developed with a conjugated secondary antibodies. However the needing of large amounts of cells is still a limit to use routinely this approach in clinical practice.[39]

A faster method employed to evaluate the intracellular or cell surface expression levels of HGF, MET and phospho-MET protein is flow cytometry. Viable cells are labeled with a fluorescein-conjugated monoclonal antibody and subsequently analyzed using a flow cytometer. Like western blotting, this method is used to evaluate the expression mostly in MM cell lines since often the amounts of primary cells are limited.[39-57] Moreover, the use of flow cytometry is already part of diagnostic procedure in some institutions and only minimal arrangements are required to include this analysis into the existing workflow.

Fluorescent in situ hybridization (FISH) can be used to determine HGF and MET gene copy number. A dual-color probe is employed and FISH results can be interpreted with different scoring systems depending on the cut-off chosen.[58-59] We performed a specific investigation of MET gene copy number in $82 \mathrm{MM}$ patients and the ratio between MET gene and control gene was used to define MET status.[46] This was the first paper investigating the presence of MET amplification in MM, although a higher number of patients are required in order to set the specific cut-off in this particular disease.

A more sensitive method to evaluate the expression levels of HGF and MET is transcript quantification by real-time reverse transcription-polymerase chain reaction (qRT-PCR). In this method, RNA is transcribed into complementary DNA (cDNA) by reverse transcriptase from total RNA or messenger RNA (mRNA) and a relative quantification is performed using a pair of unlabeled PCR primers and a probe with a reporter at the $5^{\prime}$ end, and a quencher at the $3^{\prime}$ end. For optimal results it is important that PCR primers and probes are designed carefully. Gene expression levels in real-time PCR experiments are normalized using an endogenous ("housekeeping") control gene; samples can be frozen and subsequently analized in banch in the same run on the same day to reduce costs and time and to increase accuracy.[36-51-46]

Mutational analysis of MET and HGF may be investigated with conventional capillary electrophoresis (Sanger sequencing) or next-generation sequencing technology, which is endowed with greater sensitivity. This technique has not yet been used for studies of MET 
and HGF in MM but it has been used for human solid cancers. It allows the analysis of multiple genes, the study of the MET/HGF pathway at the same time and requires limited amounts of DNA.[60]

\section{MET/HGF as targets of anti-Myeloma therapy}

The recurrence of MET/HGF abnormalities in virtually all cancers has sustained the development of several compounds aiming to interrupt this aberrant signal. Several strategies have been proposed to block the abnormal activation of the MET/HGF pathway and to interrupt the activation of intracellular cascades causing tumor aggressiveness and growth. The drugs under investigation can be divided in two large groups: biological drugs and TK inhibitors. The first are protein-based agents targeting mainly the extracellular region of the cells and can be monoclonal antibodies or antagonists of HGF. MET antagonists are truncated molecules similar to HGF; they bind MET causing only partial or minimal activation of intracellular pathway and phenotypic cell changes. Although preliminary results are interesting, their action is limited to those cells with an increase in ligand-dependent stimulation. Monoclonal Abs binds MET occupying the HGF binding site and causing blockage of phosphorylation and inhibition of downstream pathway. Similarly to MET antagonists, the mAbs are promising when abnormal Met activation is due to an increase in ligand-dependent stimulation. They are very specific and with a long half-life, but they can have suboptimal cell penetration; moreover, the production costs are high and their required intra venous administration.

On the contrary the TK inhibitors are small molecules able to interact with the intracellular part of the receptor and mainly block the kinase following HGF ligand stimulation or constitutive activation. They are usually oral and differentiate each other for the binding site or for the level of interaction with downstream pathways. One of the first TK inhibitors tested in vitro on MM cells has been PHA-665752. It is an ATP-competitive inhibitor selective for MET able to inhibit the activation of several downstream transducers, including the binding of Gab1 with MET and the HGF-mediated stimulation of Akt.[37] PHA-665752 has been shown to be able to reduce proliferation and adhesion to fibronectin both in $\mathrm{MM}$ cell lines and in primary selected PCs. The observation that it can inhibit the production of IL-11 suggested a possible role in disrupting a stimulus of bone damage.

The compounds that have been tested in vitro or in vivo in $\mathrm{MM}$ are described in detail below and are summarized in Table 2.

\section{Tivantinib (ARQ197)}

It is a highly selective non ATP-competitive inhibitor of MET. It is an oral compound that inhibits both constitutive and ligand-induced phosphorylation of MET and downstream effectors. Interestingly, ARQ197 action was observed in cell lines with different mechanisms 
of MET dysregulation such as MET gene amplification in MKN45 and ligand-dependent MET activity in MDA-MB-231. Although the role of Tivantinib as a specific Met inhibitor has been recently questioned, in vitro results have been confirmed in vivo in xenograft mouse models, with reduction in tumor growth after treatment with this compound.[61] These results suggested that the higher the levels of MET protein and MRNA, the stronger was the efficacy of ARQ197. This observation underlines how important is the pre-treatment evaluation in order to identify patient that can benefit from this treatment.[62] A Phase II trial is ongoing to test the efficacy and safety of ARQ197 in relapsed/refractory MM patients. Results have been presented on 16 patients treated with a median of 3 cycles of therapy. Grade 3-4 toxicity included fatigue (31\%), neutropenia (25\%), syncope, infection and pain (all 13\% G3). One out of 16 patients reported anal fissure, cough, fatigue, hypertension and pulmonary embolism G3. A G1-2 fatigue was reported in $94 \%$ of patients and diarrhea in 38\%. A Stable disease (SD) was reported in $4 / 11$ of evaluable patients (36\%) and it was maintained up to 11 cycles.[63]

\section{Ficlatuzumab}

It is a humanized HGF inhibitory monoclonal antibody able to neutralize the binding between HGF and MET thus blocking MET-phosphorylation and downstream intracellular cascades. After promising in vivo experiments in xenograft models, it was evaluated in a Phase I trial enrolling 41 patients with refractory solid cancer or MM. Twenty one patients received Ficlatuzumab every 14 days as monotherapy and 13 patients in combination with erlotinib (EGFR inhibitor) administered on day 1 . The G3-4 toxicity was mainly hypokalaemia (15\%), peripheral oedema $(7 \%)$, diarrhea/vomiting $(7 \%)$ and fatigue $(4 \%)$. The best overall response was SD achieved in $44 \%$ of patients with a median duration of 5.5 months. The recommended dose for a Phase II trial was identified as $20 \mathrm{mg} / \mathrm{Kg}$ every 14 days.[64]

\section{Cabozantinib (XL184)}

It is an inhibitor of several tyrosine kinases including MET, VEGFR2, RET, TYROL3 and AXL. It has been tested in a syngeneic 5TGM1 mouse model of MM with the primary aim of investigating its activity on bone lesions. MM cells were inoculated IV in the mice and the lytic lesions were detected by radiography. Interestingly, cabozantinib showed bone protective effects including reduction in total area of osteolysis, TRACP $5 \mathrm{~b}$ levels and of osteoclast number. All these effects were higher in cabozantinib-treated mice than in bortezomib-treated mice. When these drugs were administered simultaneously, an increase in OS was observed ( median OS: 43 days with bortezomib, 48 days with cabozantinib, and 55 days with both.[65]

\section{Amuvatinib (MP470)}

It is a carbothioamide able to inhibit MET, cKIT and PDGF. It is an orally available drug and has been studied in in vitro models of myeloma. It has been shown to be able to induce cell death in 6/8 of primary CD138+ cells of patients with myeloma, reducing the MET phosphorylation on Tyr $1234 / 1235$ by approximately $50 \%$. This effect was observed only in CD138+ cells and not in the CD138- fraction, which experienced only minimal cell death 
$(<10 \%)$ when cultured in presence of amuvatinib. This observation suggests that the nonmalignant bone marrow cells are not effected by amuvatinib action because they have very low level of p-MET. In the U266 myeloma cell line (expressing high level of HGF), amuvatinib was able to cause cell-cycle arrest in G1, decrease thymidine incorporation and cause increase in cell death. The cell death process was caspase-mediated and, again, was observed only in U266 cell line. When a cell line expressing very low levels of HGF and MET was used (RPMI-8226/S) only minor apoptosis induction was observed. When the downstream targets of MET were investigated, both ERK1/2 and AKT signal were inhibited.[57]

\section{SU11274}

It is a MET inhibitor tested to evaluate its ability to reduce endothelial cell growth. When ECs of MM patients were cultured with anti-HGF or anti-MET Abs, the viability and the proliferation was not impaired. However the migration activity was markedly impaired, up to $45 \%$ despite concomitant stimulation by angiogenic factors.[35] Similar results have been obtained with SU11274. Interestingly, a strong synergistic antiangiogenic effect was observed when SU11274 was added to bortezomib or lenalidomide: both inhibition of mesh areas and reduction of vessel length were doubled in the presence of of SU11274. The treatment with SU11274 also modified some angiogenesis-related cytokines, with a decrease of proangiogenic cues (SERPIN E1, CXCL16 and MCP-1) and an increase in the negative regulator SERPIN F1. These results suggest that SU11274 could interfere also with MM angiogenesis on top of its direct anti MM effects against PC.

\section{NK4}

It is an antagonist of HGF, composed of the NH2-terminal hairpin loop and the 4 kringle domains of HGF. It competes with HGF for MET binding but it does not cause any MET phosphorylation after binding. It is able to inhibit HGF-induced proliferation and to promote apoptosis of PCs both directly (acting on ERK1/2, STAT3 and AKT-1 pathways) and indirectly via angiogenesis inhibition. These observations were first obtained in cell lines and were confirmed in primary cells of MM and xenograft mouse models. Interestingly, the efficacy of NK4 has been observed also in non-HGF producing cells growing in an HGF free medium. Moreover, the histological examination of tumor tissues derived from mouse xenografts revealed that NK4 is able to reduce the vascularization around MM cells, confirming an indirect way of reducing cell growth via inhibition of angiogenesis.[66]

\section{Anti MET Nanobody:}

Nanobodies are proteins based on the smallest functional fragments of heavy chain antibodies and can be directed against different antigens. In pioneering work, Slørdahl et al demonstrated that an anti-MET nanobody is able to specifically bind MET and inhibit HGFdriven phosphorylation as well as activation of downstream signals, in particular the MAPK pathway and Akt. Moreover, the nanobody can reduce the adhesion of MM cells (adhesion is well-known mechanism of drug resistance) and can intercept the inhibitory effect of HGF on 
osteoblastogenesis. These results confirm that targeting MET/HGF can cause anti MM effects non only in malignant plasma cells but also in the surrounding cells, which contribute to bone disease and drug resistance.[67]

\section{MP0250}

It is a bispecific DARP able to inhibit both HGF and VEGF. It has been tested in vitro as monotherapy and in combination with bortezomib. Blocking HGF and VEGF is sufficient to stop a positive feedback for PC growth and to reduce bone destruction. In an orthotopic murine model with primary myeloma cells implanted in the bone marrow, treatment with MP0250 and bortezomib reduced bone lysis, as measured with micro-CT and X-ray. Albeit very preliminary, this observation suggests a possible synergistic effect of MP0250 without any additional toxicity.[68]

Beside these compounds that have been tested in MM, many other small-molecule inhibitors (such as crizotinib and golvatinib) and mAbs (such as rilotumumab and onartuzumab) are under investigation in other cancers. Overall, the compounds targeting MET/HGF have shown an acceptable toxicity profile, with side effects that are usually different from other anti myeloma drugs

\section{Conclusions}

The pathogenesis and progression of MM is very complex and only partially known. Several molecular alterations in PCs have been reported, as well as a key role of stromal cells, angiogenesis and the bone microenvironment. The survival and growth of myeloma cells are dependent on the presence of a permissive microenvironment able to support cell proliferation and protect PCs from drug toxicities. The possibility to reduce (or stop) the crosstalk between PCs and microenvironment is an appealing approach in MM.

Among several pathways, MET/HGF has been reported to be a pivotal one. Indeed, the HGF/MET axis is abnormally active in both PCs and the microenvironment and a positive feedback between these two entities has clearly been documented resulting in cell growth, invasion, bone disease and drug resistance. MET/HGF may be a promising target also to reduce angiogenesis in MM patients. MET is constitutively activated, overexpressed or stimulated by HGF in a fraction of MM patients and sustained MET stimulation has been reported in several solid and hematological cancer as a hallmark of aggressive disease. Targeting the MET/HGF pathway could break the crosstalk between myeloma cells and the surrounding environment, reducing drug resistance and increasing response to treatment. This could be of particular importance in relapsed/refractory patients where the MET/HGF system is frequently deregulated. 
Some issues need to be solved before considering this pathway a viable therapeutic target in MM. The selection of patients is still the major obstruction and the challenge will be to accurately identify patients that can benefit from such specific therapy. The results of initial clinical trials are showing that unfortunately not all patients with an abnormal MET/HGF pathway will respond to the treatment (primary resistance) highlighting the importance of biomarkers to pinpoint the patients with a higher chance of responding to MET/HGF inhibitors. Specific tests to characterize the MET/HGF pathway in each MM patient need to be developed as companion diagnostics in order to identify potential responders. This is a growing concept in medicine and oncology in particular, but becomes crucial in such a specific treatment approach as TK inhibitors.

Moreover, even responding patients will develop drug resistance along treatment (secondary resistance). The reasons for acquired resistance are manifold, but they all rely on the plasticity of oncogene activity and the presence of several crosstalks within the intracellular pathways It is thus critical to find out what are the mechanisms of acquired resistance in order to prevent, delay, or early detect them. Mutations in the target molecule is a well-known escape mechanism allowing cells to be refractory to a treatment; similarly, the activation of parallel signaling pathway enables cells to elude the consequences of target blockade. In the same vein, longitudinal monitoring of the MET/HGF pathway is mandatory to timely detect any molecular changes that can lead to resistance to therapy. All these aspects (and limitations) are particularly critical in $\mathrm{MM}$, a disease with a high degree of heterogeneity and several ways to display drug resistance. Thus, the targeting of the MET/HGF system needs to be included within a therapeutic approach that also incorporates less specific drugs such as IMiDs or proteasome inhibitors.

The timing of anti MET/HGF treatment should be carefully evaluated as well. It has been reported, as expected, that patients with relapse/refractory $(R / R) M M$ have higher levels of MET, a condition that likely predicts response to HGF/MET inhibitors; however, such patients are expected to bear tumors with a higher mutational load and with many more genetic abnormalities than early-stage patients, which might require combinatorial approaches against HGF/MET and other driver oncogenic lesions Further clinical observations are required before the best timing will be identified.

Overall, strong evidence supports the observation that abnormal HGF/MET activity has a key role in MM pathogenesis and such deregulation characterizes patients with suboptimal response to therapy and poor outcome. Promising results are coming from early clinical trials testing anti MET/HGF compounds in cancer in general, confirming their efficacy and their ability to restore chemotherapy sensitivity while displaying acceptable safety profiles.

\section{Five year view}


Targeted therapies have revolutionized the way cancer is treated, increasing significantly the life expectancy and the quality of life of patients. Due to the heterogeneity of abnormal signals, in MM the therapy is mainly based on compounds with a broad spectrum of actions. The inclusion on Immunomodulatory drugs (IMiDs) and proteasome inhibitors (PI) in all regimens led to a dramatic increase in PFS and OS in the last years, but both these classes of drugs act in a non-specific manner.

The possibility of adding a compound targeting an abnormal pathway is fascinating, but several steps are required for patients to receive clinical benefit. One critical issue to be faced in next few years will be the identification of biomarkers able to enrich for potential responders to anti MET/HGF treatment. MET/HGF is a key player in sustaining MM cell growth and in causing organ damage; however, a sensitive and reliable test to evaluate the MET/HGF pathway remains unavailable. Several techniques are under investigation and can be included in the diagnostic process, but the ability to correctly identify the patients for targeted therapy is still suboptimal.

In the last few years several compounds reached the advanced clinical phase of investigation (Phase II-III) in MM. Some of them are novel drugs of already existing class (IMiDs as pomalidomide or $\mathrm{PI}$ as carfilzomib and ixazomib) and some are new classes of drugs such as monoclonal antibodies. Several other new molecules are under investigation, with the promise to enrich the therapeutic armamentarium to treat patients with MM with specific drugs targeting abnormal pathways.

This scenario will have two major implications: the first is the possibility to inhibit abnormally active pathways critical for MM growth, the second is the need of a correct identification of patients suitable to receive this specific treatment. The first point is important in MM because several pathways can be involved in sustaining cell growth and within such pathways the $\mathrm{MET} / \mathrm{HGF}$ is one of the most important. Inhibition of this pathway has been reported to reduce cell growth and increase drug sensitivity. Several MET inhibitors are currently available and further characterization of their mechanisms of action will allow optimizing their use in myeloma patients.[69]

The real challenge will be the patient selection process, with the identification of a reliable technique to evaluate MET/HGF status and select patients who will benefit from MET inhibitors. The importance of this endeavor has been highlighted in several papers and this issue is still a barrier to widespread use of anti MET/HGF compounds in the treatment of cancer. However, the availability of several compounds targeting this pathway in virtually all cancers will speed up the process to select a reliable marker and include it into clinical trials. If the results available so far are confirmed on larger number of patients, in the next few years a new class of drugs will be available for cancer treatment in general and in particular for MM patients. 


\section{Key issues}

- MET/HGF pathway is abnormal in MM PCs and plays a role in its pathogenesis

- Abnormalities in MET/HGF increase angiogenesis and cause bone disease

- MET/HGF abnormalities usually characterize patients with poor prognosis

- Targeting MET/HGF results in cell death and can attenuate resistance to chemotherapy

- A reliable marker of sensitivity to anti MET/HGF treatment is needed to identify patients expected to benefit from anti MET therapy

- Biomarker analysis should require easy standard procedures to become a routine companion diagnostic for MM patients

- Compounds targeting the MET/HGF pathway will likely be used in combination with chemotherapy agents

- Early identification of resistance to treatment will be critical in patients treated with MET inhibitors 


\section{References:}

1. Hideshima T, Mitsiades C, Tonon G et al. Understanding multiple myeloma pathogenesis in the bone marrow to identify new therapeutic targets. Nat Rev Cancer. 2007 Aug;7(8):585-98. 2. Palumbo A, Anderson K. Multiple myeloma. N Engl J Med. 2011 Mar 17;364(11):1046-60. 3. Ghiso E, Giordano S. Targeting MET: why, where and how? Curr Opin Pharmacol 2013;13:511-8

4. Bussolino F, Di Renzo MF, Ziche M, et al. Hepatocyte growth factor is a potent angiogenic factor which stimulates endothelial cell motility and growth. J Cell Biol 1992;119:629-41

5. Derksen PW, Keehnen RM, Evers LM, et al. Cell surface proteoglycan syndecan-1 mediates hepatocyte growth factor binding and promotes Met signaling in multiple myeloma. Blood 2002;99:1405-10

6. Trusolino L, Bertotti A, Comoglio PM. MET signalling: principles and functions in development, organ regeneration and cancer. Nat Rev Mol Cell Biol 2010;11:834-48 7. Graveel CR, Tolbert D, Vande Woude GF. MET: a critical player in tumorigenesis and therapeutic target. Cold Spring Harb Perspect Biol. 2013 Jul 1;5(7).

8. Rosário M, Birchmeier W. How to make tubes: signaling by the Met receptor tyrosine kinase. Trends Cell Biol 2003;13:328-35

9. Schaeper U, Gehring NH, Fuchs KP, et al. Coupling of Gab1 to c-Met, Grb2, and Shp2 mediates biological responses. J Cell Biol 2000;149:1419-32

10. Lamorte L, Rodrigues S, Naujokas M, et al. Crk synergizes with epidermal growth factor for epithelial invasion and morphogenesis and is required for the met morphogenic program. $\mathrm{J}$ Biol Chem 2002;277:37904-11

11. Saucier C, Khoury H, Lai KM, et al. The Shc adaptor protein is critical for VEGF induction by Met/HGF and ErbB2 receptors and for early onset of tumor angiogenesis. Proc Natl Acad Sci U S A 2004;101:2345-50

12. Weimar IS, Miranda N, Muller EJ, et al. Hepatocyte growth factor/scatter factor (HGF/SF) is produced by human bone marrow stromal cells and promotes proliferation, adhesion and survival of human hematopoietic progenitor cells (CD34+). Exp Hematol 1998;26:885-94 13. Mahtouk K, Tjin EP, Spaargaren M, et al. The HGF/MET pathway as target for the treatment of multiple myeloma and B-cell lymphomas. Biochim Biophys Acta 2010;1806:20819

14. Cecchi F, Rabe DC, Bottaro DP. Targeting the HGF/Met signalling pathway in cancer. Eur J Cancer 2010;46:1260-70

15. Graziano F, Galluccio N, Lorenzini P et al. Genetic activation of the MET pathway and prognosis of patients with high-risk, radically resected gastric cancer. J Clin Oncol. 2011 Dec 20;29(36):4789-95.

16. Lennerz JK, Kwak EL, Ackerman A et al. MET amplification identifies a small and aggressive subgroup of esophagogastric adenocarcinoma with evidence of responsiveness to crizotinib. J Clin Oncol. 2011 Dec 20;29(36):4803-10.

17. Mariani M, McHugh M, Petrillo $M$ et al. HGF/c-Met axis drives cancer aggressiveness in the neo-adjuvant setting of ovarian cancer. Oncotarget. 2014 Jul 15;5(13):4855-67.

18. Sierra JR, Tsao MS. c-MET as a potential therapeutic target and biomarker in cancer. Ther Adv Med Oncol 2011;3:S21-35

19. Prat M, Oltolina F and Basilico C. Monoclonal Antibodies against the MET/HGF Receptor and Its Ligand: Multitask Tools with Applications from Basic Research to Therapy.

Biomedicines 2014;2:359-383 
20. Engelman JA, Zejnullahu K, Mitsudomi T, et al. MET amplification leads to gefitinib resistance in lung cancer by activating ERBB3 signaling. Science 2007;316:1039-43

21. Gherardi E, Birchmeier W, Birchmeier C, et al. Targeting MET in cancer: rationale and rogress. Nat Rev Cancer 2012;12:89-103

22. Liu L, Zeng W, Wortinger MA, et al. LY2875358, a neutralizing and internalizing anti-MET bivalent antibody, inhibits HGF-dependent and HGF-independent MET activation and tumor growth. Clin Cancer Res 2014;20:6059-70

23. Acunzo M, Romano G, Palmieri D, et al. Cross-talk between MET and EGFR in non-small cell lung cancer involves miR-27a and Sprouty2. Proc Natl Acad Sci U S A 2013;110:8573-78 24. Pennacchietti S, Michieli P, Galluzzo M, et al. Hypoxia promotes invasive growth by transcriptional activation of the met protooncogene. Cancer Cell 2003;3:347-61

25. Röllig C, Knop S, Bornhäuser M. Multiple myeloma. Lancet. 2014 Dec 22. pii: S01406736(14)60493-1.

26. Rocci A, Hofmeister CC, Pichiorri F. The potential of miRNAs as biomarkers for multiple myeloma. Expert Rev Mol Diagn. 2014 Nov;14(8):947-59.

27. Gambella M, Rocci A, Passera R et al. High XBP1 expression is a marker of better outcome in multiple myeloma patients treated with bortezomib. Haematologica. 2014 Feb;99(2):e14-6.

28. Börset M, Hjorth-Hansen H, Seidel C, et al. Hepatocyte growth factor and its receptor Cmet in multiple myeloma. Blood 1996;88:3998-4004

29. Seidel C, Lenhoff $S$, Brabrand S, et al. Hepatocyte growth factor in myeloma patients treated with high-dose chemotherapy. Br J Haematol 2002;119:672-76

30. Wader KF, Fagerli UM, Holt RU, et al. Elevated serum concentrations of activated hepatocyte growth factor activator in patients with multiple myeloma. Eur $\mathrm{J}$ Haematol 2008;81:380-83

31. Tjin EP, Derksen PW, Kataoka H, et al. Multiple myeloma cells catalyze hepatocyte growth factor (HGF) activation by secreting the serine protease HGF-activator. Blood 2004;104:2172-75

32. Wader KF, Fagerli UM, Børset M, et al. Immunohistochemical analysis of hepatocyte growth factor and c-Met in plasma cell disease. Histopathology 2012;60:443-51

33. Rajkumar SV, Mesa RA, Fonseca R, et al. Bone marrow angiogenesis in 400 patients with monoclonal gammopathy of undetermined significance, multiple myeloma, and primary amyloidosis. Clin Cancer Res 2002;8:2210-16

34. Zhang YW, Su Y, Volpert OV, et al. Hepatocyte growth factor/scatter factor mediates angiogenesis through positive VEGF and negative thrombospondin 1 regulation. Proc Natl Acad Sci U S A 2003;100:12718-23

35. Ferrucci A, Moschetta M, Frassanito MA, et al. A HGF/cMET autocrine loop is operative in multiple myeloma bone marrow endothelial cells and may represent a novel therapeutic target. Clin Cancer Res 2014;20:5796-807

36. Stellrecht CM, Phillip CJ, Cervantes-Gomez F, et al. Multiple myeloma cell killing by depletion of the MET receptor tyrosine kinase. Cancer Res 2007;67:9913-20

37. Hov H, Holt RU, Rø TB, et al. A selective c-met inhibitor blocks an autocrine hepatocyte growth factor growth loop in ANBL-6 cells and prevents migration and adhesion of myeloma cells. Clin Cancer Res 2004;10:6686-94

38. Holt RU, Fagerli UM, Baykov V, et al. Hepatocyte growth factor promotes migration of human myeloma cells. Haematologica 2008;93:619-22

39. Hov H, Tian E, Holien T, et al. c-Met signaling promotes IL-6-induced myeloma cell proliferation. Eur J Haematol 2009;82:277-87 
40. Manier S, Sacco A, Leleu X, et al. Bone marrow microenvironment in multiple myeloma progression. J Biomed Biotechnol 2012;2012:157496

41. Standal T, Abildgaard N, Fagerli UM, et al. HGF inhibits BMP-induced osteoblastogenesis: possible implications for the bone disease of multiple myeloma. Blood 2007;109:3024-30

42. Kristensen IB, Christensen JH, Lyng MB, et al. Hepatocyte growth factor pathway upregulation in the bone marrow microenvironment in multiple myeloma is associated with lytic bone disease. Br J Haematol 2013;161:373-82

43. Grano M, Galimi F, Zambonin G, et al. Hepatocyte growth factor is a coupling factor for osteoclasts and osteoblasts in vitro. Proc Natl Acad Sci U S A 1996;93:7644-48 44. Hjertner O, Torgersen ML, Seidel C, et al. Hepatocyte growth factor (HGF) induces interleukin-11 secretion from osteoblasts: a possible role for HGF in myeloma-associated osteolytic bone disease. Blood 1999;94:3883-88

45. Seidel C1, Børset M, Turesson I, et al. Elevated serum concentrations of hepatocyte growth factor in patients with multiple myeloma. The Nordic Myeloma Study Group. Blood 1998;91:806-12

46. Rocci A, Gambella M, Aschero S, et al. MET dysregulation is a hallmark of aggressive disease in multiple myeloma patients. Br J Haematol 2014;164:841-50

47. Ludek $P$, Hana $S$, Zdenek $A$, et al. Treatment response to bortezomib in multiple myeloma correlates with plasma hepatocyte growth factor concentration and bone marrow thrombospondin concentration. Eur J Haematol 2010;84:332-36

48. Wader KF, Fagerli UM, Holt RU, et al. Soluble c-Met in serum of patients with multiple myeloma: correlation with clinical parameters. Eur J Haematol 2011;87:394-99

49. Bardella $C$, Dettori $D$, Olivero $M$, et al. The therapeutic potential of hepatocyte growth factor to sensitize ovarian cancer cells to cisplatin and paclitaxel in vivo. Clin Cancer Res 2007;13:2191-98

50. Yakes FM, Chen J, Tan J, et al. Cabozantinib (XL184), a novel MET and VEGFR2 inhibitor, simultaneously suppresses metastasis, angiogenesis, and tumor growth. Mol Cancer Ther 2011;10:2298-308

51. Moschetta M, Basile A, Ferrucci A, et al. Novel targeting of phospho-cMET overcomes drug resistance and induces antitumor activity in multiple myeloma. Clin Cancer Res 2013;19:4371-82

52. Ma PC, Jagadeeswaran R, Jagadeesh $S$, et al. Functional expression and mutations of cMet and its therapeutic inhibition with SU11274 and small interfering RNA in non-small cell lung cancer. Cancer Res 2005;65:1479-88

53. Que W, Chen J, Chuang M, et al. Knockdown of c-Met enhances sensitivity to bortezomib in human multiple myeloma U266 cells via inhibiting Akt/mTOR activity. APMIS

2012;120:195-203

54. Que W, Chen J. Knockdown of c-Met inhibits cell proliferation and invasion and increases chemosensitivity to doxorubicin in human multiple myeloma U266 cells in vitro. Mol Med Rep 2011;4:343-49

55. Wang H, Baladandayuthapani V, Lin HY, et al. Mucin 20 (MUC20) Modulates Proteasome Assembly Chaperones through the c-MET Pathway and Is a Biomarker of Proteasome Inhibitor Sensitivity in Myeloma. San Francisco: 57th ASH Annual Meeting \& Exposition, 2014 56. Rampa C, Tian E, Våtsveen TK, et al. Identification of the source of elevated hepatocyte growth factor levels in multiple myeloma patients. Biomark Res 2014;2:8. 
57. Phillip CJ, Zaman S, Shentu S, et al. Targeting MET kinase with the small-molecule inhibitor amuvatinib induces cytotoxicity in primary myeloma cells and cell lines. J Hematol Oncol 2013;6:92

58. Minuti G, Cappuzzo F, Duchnowska R, et al. Increased MET and HGF gene copy numbers are associated with trastuzumab failure in HER2-positive metastatic breast cancer. Br J Cancer 2012;107:793-99

59. Koeppen H, Yu W, Zha J, et al. Biomarker analyses from a placebo-controlled phase II study evaluating erlotinib \pm onartuzumab in advanced non-small cell lung cancer: MET expression levels are predictive of patient benefit. Clin Cancer Res 2014;20:4488-98. 60. Ma PC, Tretiakova MS, MacKinnon AC, et al. Expression and mutational analysis of MET in human solid cancers. Genes Chromosomes Cancer 2008;47:1025-37

61. Michieli P, Basilico C, Pennacchietti S. Tivantinib (ARQ197) displays cytotoxic activity that is independent of its ability to bind MET--response. Clin Cancer Res. 2013 Aug $1 ; 19(15): 4291$.

62. Munshi N, Jeay S, Li Y, et al. ARQ 197, a novel and selective inhibitor of the human c-Met receptor tyrosine kinase with antitumor activity. Mol Cancer Ther 2010;9:1544-53

63. Orlowski RZ, Zaman S, Thomas SK, et al. Phase II Study Of The c-MET Inhibitor ARQ 197 (Tivantinib) In Patients With Relapsed Or Relapsed/Refractory Multiple Myeloma (RRMM). New Orleans, 56th ASH Annual Meeting \& Exposition, 2013

64. Patnaik A, Weiss GJ, Papadopoulos KP et al. Phase I ficlatuzumab monotherapy or with erlotinib for refractory advanced solid tumours and multiple myeloma. $\mathrm{Br} \mathrm{J}$ Cancer. $2014 \mathrm{Jul}$ 15;111(2):272-80.

65. Aftab DT, Suominen MI, Clary DO, et al. Effects of Cabozantinib Alone and in Combination with Bortezomib in the 5TGM1 Murine Multiple Myeloma Model. San Francisco: 57th ASH Annual Meeting \& Exposition, 2014

66. Du W, Hattori Y, Yamada T, et al. NK4, an antagonist of hepatocyte growth factor (HGF), inhibits growth of multiple myeloma cells: molecular targeting of angiogenic growth factor. Blood 2007;109:3042-49

67. Slørdahl TS, Denayer T, Moen SH, et al. Anti-c-MET Nanobody - a new potential drug in multiple myeloma treatment. Eur J Haematol 2013;91:399-410

68. Fiedler W, Sessa C, Gianni L, et al. First-in-human phase I study of CetuGEX, a novel anti-EGFR monoclonal antibody ( $\mathrm{mAb}$ ) with optimized glycosylation and antibody dependent cellular cytotoxicity. Chicago: ASCO Annual Meeting, 2013

69. Zhu K, Kong X, Zhao D, et al. c-MET kinase inhibitors: a patent review (2011 - 2013). Expert Opin Ther Pat 2014;24:217-30 
Table 1: Techniques to evaluate MET/HGF

\begin{tabular}{|c|c|c|}
\hline Methods & Advantages & Disadvantages \\
\hline ELISA & $\begin{array}{l}\text { - Use of stored samples } \\
\text { - High-throughput } \\
\text { - Less labor intensive }\end{array}$ & $\begin{array}{l}\text { - Requires a standard curve } \\
\text { - Detection of only one target }\end{array}$ \\
\hline IMMUNOHISTOCHEMISTRY & $\begin{array}{l}\text { - Not requires viable cells } \\
\text { - Possible to detect two targets } \\
\text { simultaneously }\end{array}$ & $\begin{array}{l}\text { - Non specific background } \\
\text { - Labor intensive }\end{array}$ \\
\hline WESTERN BLOTTING & $\begin{array}{l}\text { - Not requires viable cells } \\
\text { - Detection of several targets }\end{array}$ & $\begin{array}{l}\text { - Requires high skill level of the } \\
\text { experimenter } \\
\text { - Labor intensive }\end{array}$ \\
\hline FLOW CYTOMETRY & $\begin{array}{l}\text { - Measures several antigens } \\
\text { simultaneously } \\
\text { - High sensibility }\end{array}$ & $\begin{array}{l}\text { - Requires high skill level of the } \\
\text { experimenter } \\
\text { - Requires viable cells } \\
\text { - Requires an appropriate amount } \\
\text { of cells }\end{array}$ \\
\hline FISH & - Not requires viable cells & $\begin{array}{l}\text { - Labor intensive } \\
\text { - Different scoring systems }\end{array}$ \\
\hline & $\begin{array}{l}\cdot \text { High sensibility } \\
\cdot \text { High-throughput }\end{array}$ & - Primers and probes design \\
\hline NGS & $\begin{array}{l}\text { - Requires low amounts of DNA } \\
\text { - Analysis of multiple genes } \\
\text { - High sensibility } \\
\text { - High-throughput }\end{array}$ & $\begin{array}{l}\text { - Requires skill bioinformatics } \\
\text { - High cost }\end{array}$ \\
\hline
\end{tabular}

ELISA= enzyme-linked immunosorbent assay; FISH= fluorescent in situ hybridization; qRT-PCR= realtime reverse transcription-polymerase chain reaction; NGS= next-generation sequencing 
Table 2: anti MET/HGF compounds

\begin{tabular}{|c|c|c|c|c|c|}
\hline Drug Name & $\begin{array}{c}\text { Mechanism } \\
\text { of action }\end{array}$ & Target & $\begin{array}{c}\text { Phase of } \\
\text { investigation }\end{array}$ & Results & Reference \\
\hline PHA-665752 & TK inhibitor & MET & $\begin{array}{l}\text { preclinical } \\
\text { (cell lines, } \\
\text { primary cells) }\end{array}$ & $\begin{array}{c}\text { inhibition of MET } \\
\text { phosphorilation and } \\
\text { downstream signalling. } \\
\text { Blockage of proliferation } \\
\text { in primary myeloma cells. }\end{array}$ & 37 \\
\hline $\begin{array}{l}\text { Tivantinib } \\
\text { (ARQ197) }\end{array}$ & TK inhibitor & MET & Phase II & $\begin{array}{c}16 \text { R/R MM patients. } \\
\text { G3-4 toxicity: neutropenia, } \\
\text { syncope, infection, pain. } \\
\text { Response: SD in } 4 / 11 \\
\text { patients }\end{array}$ & 63 \\
\hline Ficlatuzumab & $\begin{array}{c}\text { HGF } \\
\text { antagonist }\end{array}$ & HGF & Phase I & $\begin{array}{l}41 \text { patients ( } 4 \text { with MM). } \\
\text { G3-4 toxicity: fatigue, } \\
\text { peripheral oedema, } \\
\text { diarrhoea, vomiting, } \\
\text { hypokalaemia. } \\
\text { Reponse: SD in 12/24 } \\
\text { patients }\end{array}$ & 64 \\
\hline $\begin{array}{l}\text { Cabozantinib } \\
\text { (XL184) }\end{array}$ & TK inhibitor & $\begin{array}{l}\text { MET, } \\
\text { VEGFR2, } \\
\text { RET, TAM } \\
\text { family }\end{array}$ & $\begin{array}{c}\text { Preclinical } \\
\text { (xenograft } \\
\text { mouse model) }\end{array}$ & $\begin{array}{l}\text { bone protection action } \\
\text { and anti tumor effects. Its } \\
\text { activity is synergistic with } \\
\text { bortezomib }\end{array}$ & 65 \\
\hline $\begin{array}{l}\text { Amuvatinib } \\
\text { (MP470) }\end{array}$ & TK inhibitor & $\begin{array}{l}\text { MET, cKIT, } \\
\text { PDGFR }\end{array}$ & $\begin{array}{c}\text { preclinical } \\
\text { (cell lines, } \\
\text { primary cells) }\end{array}$ & $\begin{array}{c}\text { inhibition of MET } \\
\text { phosphorilation, induction } \\
\text { of apoptosis and growth } \\
\text { inhibiton in primary } \\
\text { myeloma cells }\end{array}$ & 57 \\
\hline SU11274 & TK inhibitor & MET & $\begin{array}{l}\text { preclinical } \\
\text { (cell lines, } \\
\text { primary cells, } \\
\text { mouse model) }\end{array}$ & $\begin{array}{l}\text { inhibition of endotelial } \\
\text { cells proliferation, } \\
\text { reduction of angiogenesis- } \\
\text { related cytokines, } \\
\text { synergistic effect with } \\
\text { bortezomib }\end{array}$ & 35 \\
\hline NK4 & $\begin{array}{c}\text { HGF } \\
\text { antagonist }\end{array}$ & HGF & $\begin{array}{l}\text { preclinical } \\
\text { (cell lines, } \\
\text { primary cells, } \\
\text { mouse model) }\end{array}$ & $\begin{array}{l}\text { inhibit growth of myeloma } \\
\text { cells in vitro and in } \\
\text { xenograft mouse model. } \\
\text { Reduction of } \\
\text { angiogenesis substained } \\
\text { by HGF. }\end{array}$ & 66 \\
\hline $\begin{array}{l}\text { Anti MET } \\
\text { Nanobody }\end{array}$ & anti-MET Abs & MET & $\begin{array}{c}\text { preclinical } \\
\text { (cell lines, } \\
\text { primary cells) }\end{array}$ & $\begin{array}{l}\text { inhibition of migration, } \\
\text { adhesison and } \\
\text { proliferation of myeloma } \\
\text { cells, blockage of HGF- } \\
\text { mediated inhibition of } \\
\text { osteoblastogenesis } \\
\end{array}$ & 67 \\
\hline
\end{tabular}


Fig 1: MET/HGF pathway and druggable targets

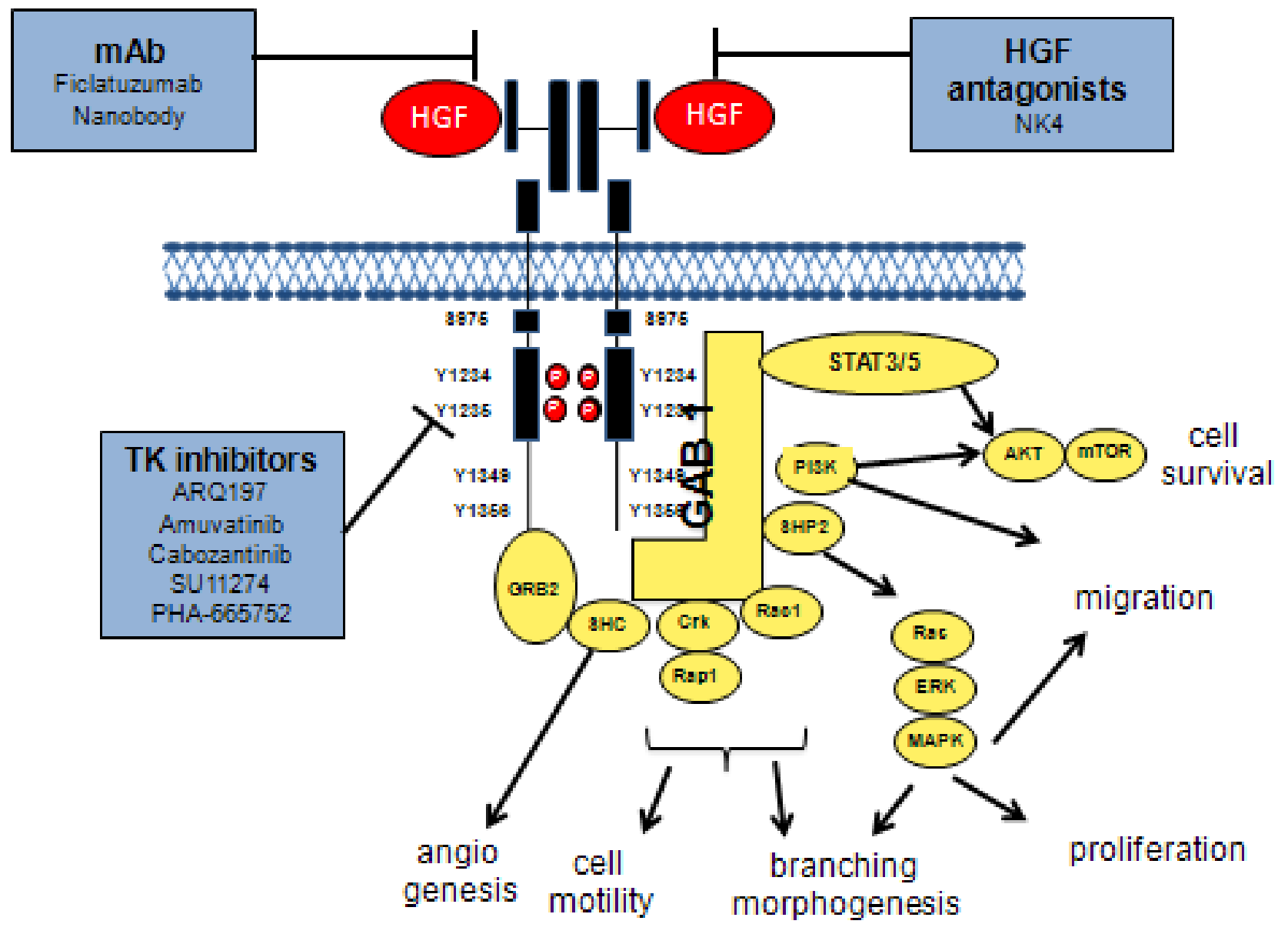


Fig 2: role of MET/HGF in the relationship between plasma cells - bone cells and Angiogenesis

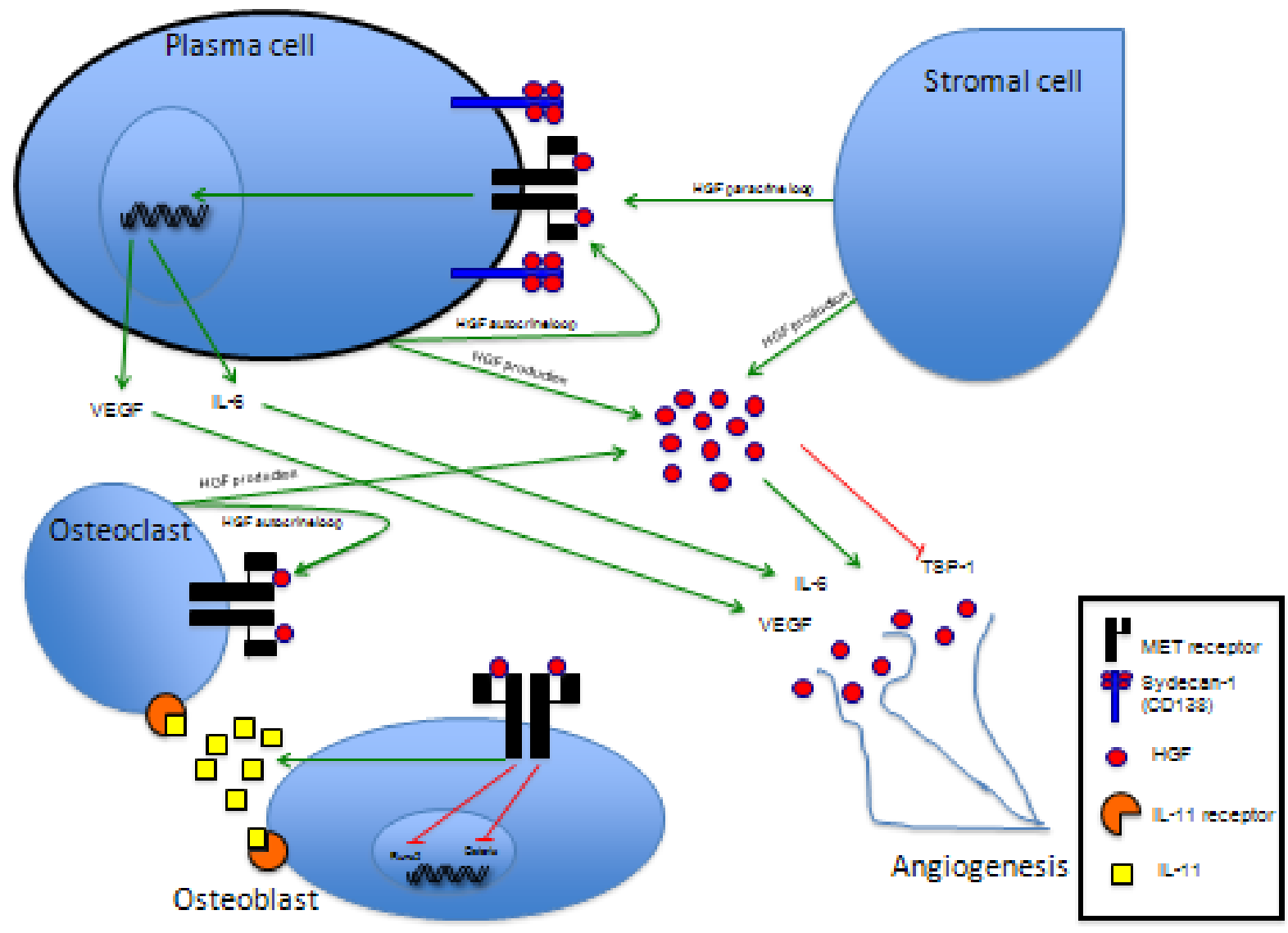

